\title{
Describing and Matching 2D Shapes by Their Points of Mutual Symmetry*
}

\author{
Arjan Kuijper ${ }^{1}$ and Ole Fogh Olsen ${ }^{2}$ \\ ${ }^{1}$ RICAM, Linz, Austria \\ arjan.kuijper@oeaw.ac.at \\ ${ }^{2}$ IT-University of Copenhagen, Denmark \\ fogh@itu.dk
}

\begin{abstract}
A novel shape descriptor is introduced. It groups pairs of points that share a geometrical property that is based on their mutual symmetry. The descriptor is visualized as a diagonally symmetric diagram with binary valued regions. This diagram is a fingerprint of global symmetry between pairs of points along the shape. The descriptive power of the method is tested on a well-known shape data base containing several classes of shapes and partially occluded shapes. First tests with simple, elementary matching algorithms show good results.
\end{abstract}

\section{Introduction}

One method to describe $2 \mathrm{D}$ objects is by their outlines, or shapes. The complicated task of comparing objects then changes to comparing shapes. With a suitable representation, this task can be simplified. Several representations of shapes have been investigated in order to be able to perform this comparison efficiently and effectively. One of the earliest representations is Blum's biologically motivated skeleton [1. As Kimia points out 2, there is evidence that humans use this type of representation.

Research on skeleton-based methods has been carried out in enormous extent ever since, see e.g. [3, 4]. The Shock Graph approach [5] has lead to a shape descriptor that can perform the comparison task very well [4, 6, 7. This method depends on results obtained from the so-called Symmetry Set [8, 9], a super set of the Medial Axis. In these cases, the shape is probed with circles tangent to it at at least two places. The Symmetry Set is obtained as the centres of all these circles, while the Medial Axis is the sub set containing only maximal circles.

From the field of robotics, probing shapes is also of interest. Blake et al. 10, 11 describe a grasping method by the set of points that are pair wise parallel. At such a pair a parallel jaw gripper can grasp the object. These points form

* This work was supported by the European Union project DSSCV (IST-2001-35443). A.K. acknowledges for funding the Johann Radon Institute (RICAM) of the ÖAW, the Austrian Science Funding Agencies FWF, through the Wittgenstein Award (2000) of Peter Markowich, and FFG, through the Research \& Development Project 'Analyse Digitaler Bilder mit Methoden der Differenzialgleichungen'. 
the union of the Symmetry Set and a set they called anti-Symmetry Set, as it is closely related to the symmetry set 12 .

In this work, we combine the ideas of these two fields of shape analysis by investigating the set of pairs of points at which a circle is tangent to the shape. We do not consider the centre of the circle, but the combination of the two points. A geometric method is given to derive the pairs of points, based on a zero crossing argument. Therefore, to each pair of points a signed value can be assigned, yielding a matrix of values $(-1,0,1)$.

This matrix is then used as a shape descriptor. Its properties and allowed changes follow directly from the Symmetry Set, just as in the Shock Graph method. Next, a simple comparison algorithm is introduced to perform the task of object comparison. For this purpose, the two matrices for each pair of objects are set to equal dimensions and the normalised inner product is taken as equivalence measure. This procedure is tested on two data bases containing objects in different classes, where some objects are occluded or noisy. Given the simplicity of the algorithm, results are promising and main erroneous results are due to the algorithm, showing the potential power of the representation.

\section{Problem Framework and Definitions}

The Medial Axis can be defined as the closure of the loci of the maximal circles tangent to a shape (see e.g. [9]). This somewhat abstract formulation can be made clear by investigation of Figure 17. A circle with radius $r$ is tangent to a shape at two points. The unit length normal vectors $\left(N_{1}\right.$ and $\left.N_{2}\right)$ of the circle and the shape coincide. The centre of the circle is a Medial Axis point, that is found by multiplying each normal vector with $-r$ and taking the tangency point as tail of the vector $-r N_{i}$. As there are for each point several combinations satisfying this tangency argument 1 , the set is taken for with $-r$ is maximal, i.e. the set with the smallest radius.

The two points can be found using geometrical arguments [8], see Fig. 1]b. Take an arbitrary origin point and let $p_{1}$ and $p_{2}$ be vectors pointing to the two locations of tangency. Then $p_{1}-p_{2}$ is a vector pointing from one tangency point to the other. From the construction of the circle as described before, the vector $-r N_{1}+r N_{2}$ (and when normal vectors are pointing inward and outward $\left.-r N_{1}-r N_{2}\right)$ is parallel to $p_{1}-p_{2}$. Consequently $\left(p_{1}-p_{2}\right) \cdot\left(N_{1} \pm N_{2}\right)=0$ for these two points. Let a shape be continuously parameterised then for each point $p$ several points $q_{j}$ can be found for which

$$
\left(p-q_{j}\right) \cdot\left(N(p) \pm N\left(q_{j}\right)\right)=0
$$

where $N($.$) denotes the normal vector. Note that if the normal vectors are par-$ allel, the inner product is zero as well. Such points are the anti-Symmetry Set points described by Blake et al. [10,11] for the parallel jaw gripper. If the shape

${ }^{1}$ It can be shown that for each point there are at least two other points [8]. Constellations with tangency normal vectors pointing inside and outside can occur [9]. 


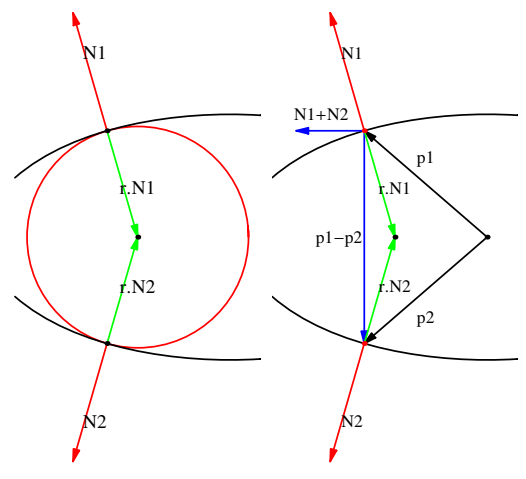

Fig. 1. a) A pair of tangency points that gives rise to a Medial Axis point. b) The constellation of position and normal vectors is special at such points.

is parameterised by $N$ points $\left(p_{1}, p_{2}, \ldots, p_{N}\right)$, then the tangency pairs are found as the zero crossings of Eq. 11. To find these zero crossings, it suffices to look at the square sign of inner product diagram $P(i, j)$ of the signed values of Eq1

$$
P(i, j)=\operatorname{sign}\left[\left(p_{i}-p_{j}\right) \cdot\left(N_{i} \pm N_{j}\right)\right]
$$

In Figure 2 a fish shape is shown, together with its sign of inner product diagram. When actual zero crossings are computed, i.e. when the boundaries of the regions in such a diagram are taken, one obtains a so-called pre-Symmetry Set that is used to derive the distinct branches of the Symmetry Set [8, 13]. The possible changes of these boundaries when the shape changes, are known [14] and relate to the possible changes of the Medial Axis [9].

Changes in the shape lead to movement of the boundaries and therefore to changes of areas. Topological changes fall apart into two classes: Firstly, boundaries can meet and establish a different connection when a white (or black) region
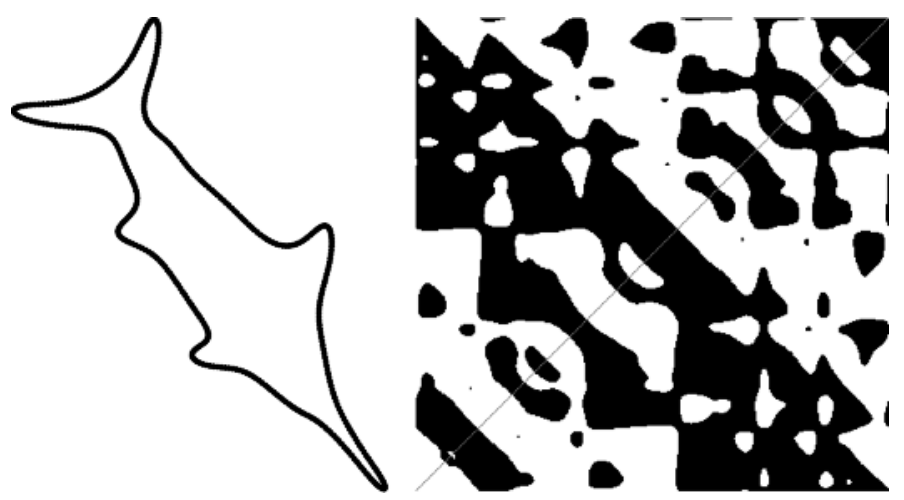

Fig. 2. a) A fish shape. b) Sign of inner product diagram for the fish shape. 
is locally split into two parts. Secondly, regions can be annihilated or created, either on the diagonal or pair-wise off-diagonal. Other possible changes of the Symmetry Set do not lead to topological changes.

As may be clear from Eq. 2, the diagram is symmetric in the diagonal. It can be identified with the shape, just as (by definition) the axes of the diagram. The values on the diagonal equal zero, as these points cannot be evaluated in Eq. 1. Second, on all other point combinations it is non-generic to encounter exactly a zero-crossing, so either a positive or a negative sign is obtained.

\section{Sign of Inner Product Diagram Based Matching}

The task of comparing objects has now become the task of comparing diagrams. If the parameterisations of two shapes consist of the same amount of points $n$, the corresponding sign of inner product diagrams can be multiplied element wise with each other. If the shapes are identical and the parameterisations are equal, this inner product equals $n(n-1)$, since the diagonal consists of $n$ points.

If the parameterisations are taken at a different starting position, so that $p_{i}=q_{i+\alpha}$, rotated version of the sign of inner product diagram should be taken into account. This rotation takes place in horizontal and vertical directions simultaneously, as $P(i, j)=Q(i+\alpha, j+\alpha)$, values taken modulo $n$. So to validate each possible starting position, $n$ instances need to be compared.

Finally, the number of points for both shapes need not be equal. If the difference is $m$ rows (and columns), a method must be chosen that removes $m$ rows and columns. One choice is to remove them equally spread over the largest sign of inner product diagram. This relates to removing a set of equidistant points along shape with the largest number of points. It can be regarded as a re-parameterisation of the shape with the largest number of points.

Now let two shapes $S_{1}, S_{2}$ be parameterised with $n_{1}$ and $n_{2}$ points. Assume without loss of generality $n_{1} \leq n_{2}$. The sign of inner product diagram of $S_{2}$ is denoted by $P_{1}$. Let $n=n_{1}$ and $m=n_{2}-n_{1}$. Build $P_{2}$ by removing each $\left(\frac{m}{n_{2}}\right)^{t h}$ row and column of the sign of inner product diagram of $S_{2}$. Let $P_{1}^{r}$ denote the sign of inner product diagram $P_{1}$ considered with as starting position point $r$ on the shape, i.e. $P_{1}$ with its first $r-1$ columns and rows transferred to positions $n+1, \ldots, n+r-1$ :

$$
P_{1}^{r}(i, j)=P_{1}(i-r+1, j-r+1)
$$

where values are taken modulo $n$. This matches the shapes regardless of begin position of the parameterisations. Then the matching $D\left(P_{1}, P_{2}\right)$ between $S_{1}, S_{2}$ is set as

$$
D\left(S_{1}, S_{2}\right)=\max _{r}\left(D\left(S_{1}^{r}, S_{2}\right)\right)
$$

with

$$
D\left(S_{1}^{r}, S_{2}\right)=\frac{\sum_{i=1}^{n} \sum_{j=1}^{n} P_{1}^{r}(i, j) P_{2}(i, j)}{n(n-1)}-\frac{m}{2 n_{2}}
$$


The first term in Eq. 4 denotes the weighted equality of the two sign of inner product diagram $P_{1}^{r}, P_{2}$. Perfect match is given by 1 , while a complete mismatch equals -1 and a random match 0 . The second term penalises the difference in number of points in a parameterisation, as this difference is ignored in the first term by construction. Adding this penalty is motivated by the way the shapes are obtained, viz. as the outlines of standardised binary images. Therefore, the number of points relates to the complexity of the shape.

\section{Data Base Matching}

As first test set 41 shapes from an online data base are taken 2 . They form three classes: fishes, planes, and tools. Some fishes and planes are artificially drawn, and form inter class instances. The results of matching all shapes with each
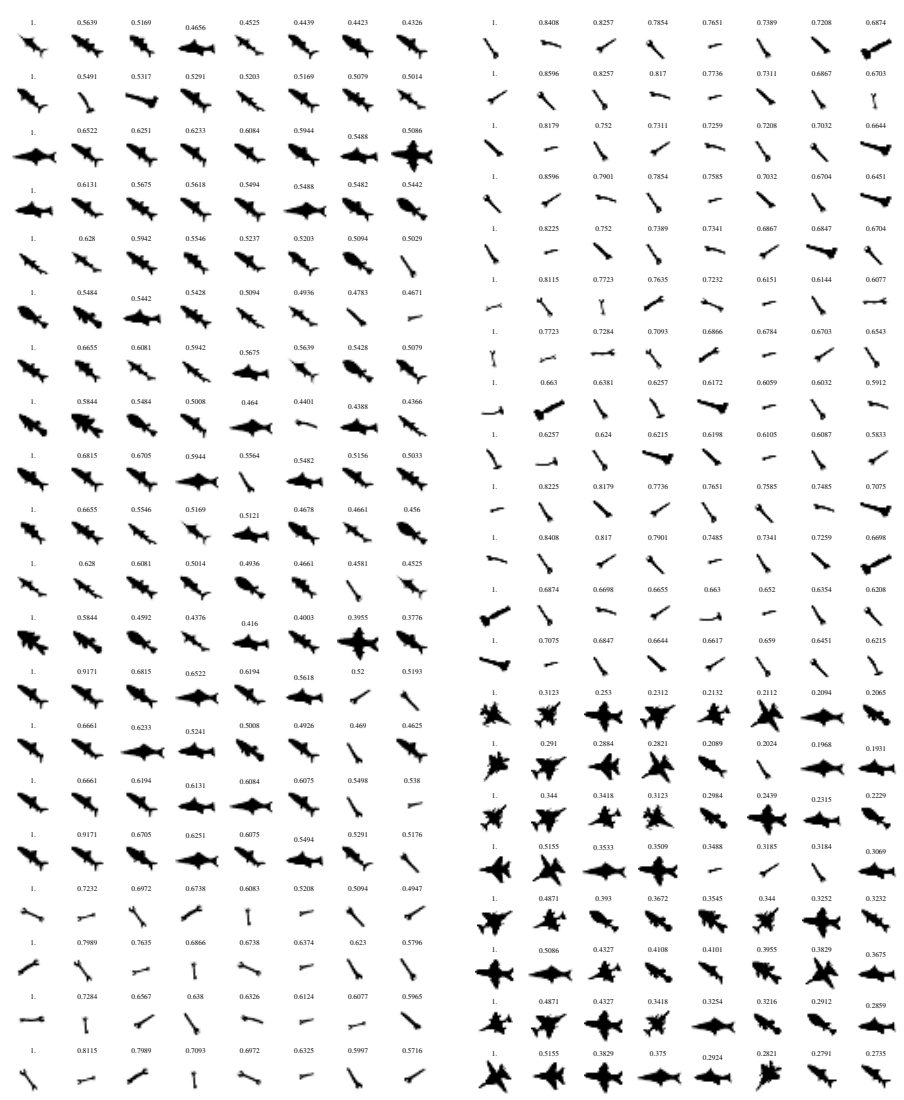

Fig. 3. Matching of fishes, tools, and planes

${ }^{2}$ http://www.lems.brown.edu/vision/researchAreas/SIID/ 
other can be seen in Figure 3. For each shape, the best eight matches are shown: The first column has score zero, as each shape matches to itself without difference. The second column gives the second best match, etc.

The matching is consistent with [15], where this database is introduced. One can see, for instance, that tools match to tools, and that the wrenches and double wrenches match to the correct set. The erroneous matches - the appearances of shapes of a different class - occur at a match $D=.5$ or less. These errors can

Table 1. Score of inter-class matches

\begin{tabular}{|c|c|}
\hline class & score \\
\hline 1 & $11,11,11,11,11,11,11,8,6,7,1$ \\
2 & $11,11,9,10,8,6,8,5,4,5,2$ \\
3 & $11,10,10,10,10,10,9,8,9,7,2$ \\
4 & $11,11,11,10,10,8,9,10,7,6,3$ \\
5 & $11,10,9,9,7,8,1,2,0,3,6$ \\
6 & $11,11,11,11,11,11,11,11,11,11,11$ \\
7 & $11,11,11,10,10,8,6,8,2,3,3$ \\
8 & $11,10,10,11,9,9,9,8,7,3,2$ \\
9 & $11,11,11,11,11,11,11,11,11,11,11$ \\
\hline
\end{tabular}

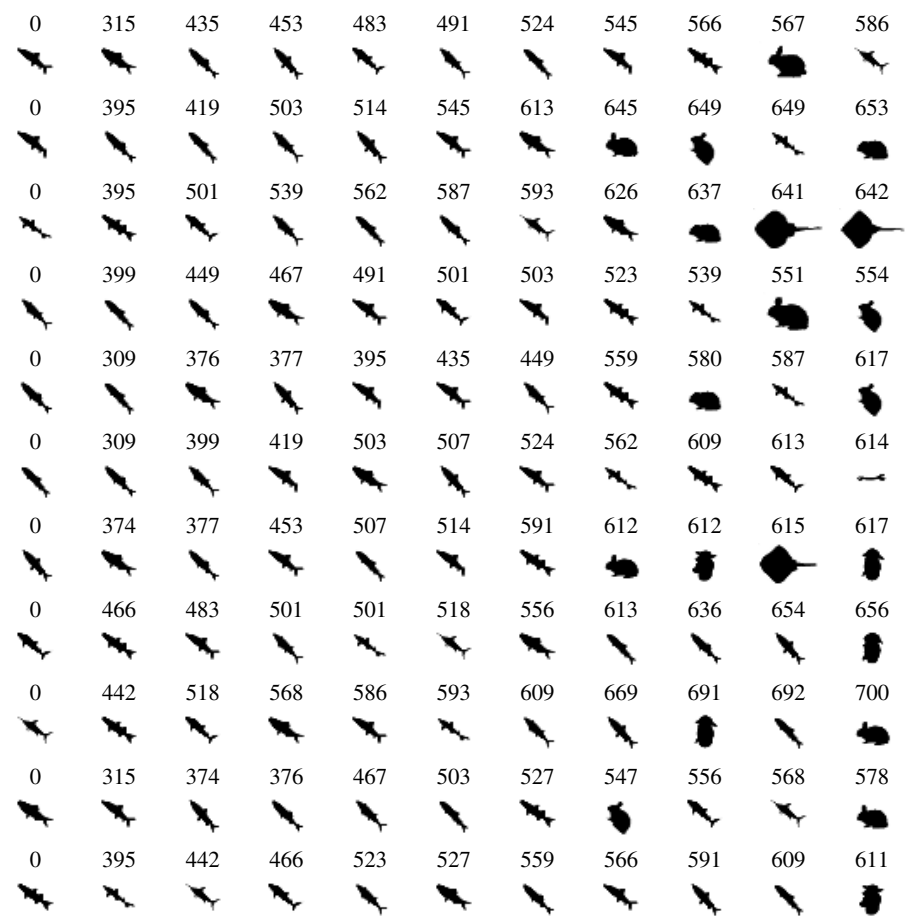

Fig. 4. Class 1 


\begin{tabular}{|c|c|c|c|c|c|c|c|c|c|c|}
\hline 0 & 68 & 576 & 616 & 700 & 737 & 754 & 770 & 781 & 795 & 805 \\
\hline$\pi$ & $r$ & $h$ & m & $n$ & 7 & $n$ & $\star$ & $\star$ & - & $T$ \\
\hline 0 & 68 & 596 & 601 & 686 & 743 & 752 & 758 & 772 & 799 & 809 \\
\hline$r$ & $\pi$ & $m$ & $h$ & $n$ & $m$ & $r$ & 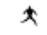 & $\star$ & $m$ & 1 \\
\hline 0 & 322 & 467 & 638 & 650 & 686 & 700 & 726 & 762 & 790 & 792 \\
\hline$n$ & $r$ & $\pi$ & $\pi$ & $m$ & $\pi$ & $\pi$ & $h$ & $\pi$ & $\star$ & $\pi$ \\
\hline 0 & 322 & 532 & 717 & 741 & 752 & 754 & 760 & 764 & 766 & 779 \\
\hline$n$ & $n$ & $m$ & $m$ & $\star$ & $r$ & $\pi$ & $\star$ & $\star$ & $\star$ & $t$ \\
\hline 0 & 543 & 548 & 638 & 709 & 743 & 783 & 787 & 795 & 796 & 797 \\
\hline$m$ & $n$ & $m$ & $n$ & $n$ & 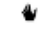 & $r$ & $*$ & $\pi$ & 5 & $m$ \\
\hline 0 & 467 & 532 & 548 & 708 & 721 & 724 & 737 & 743 & 752 & 758 \\
\hline 7 & $n$ & $n$ & $\pi$ & 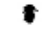 & $h$ & $t$ & $\pi$ & $n$ & $n$ & $v$ \\
\hline 0 & 576 & 601 & 657 & 709 & 719 & 721 & 726 & 761 & 774 & 806 \\
\hline$n$ & $\pi$ & $n$ & $n$ & $m$ & 5 & $\pi$ & $n$ & $*$ & 1 & $m$ \\
\hline 0 & 523 & 737 & 769 & 798 & 809 & 848 & 848 & 854 & 876 & 876 \\
\hline$\pi$ & $M$ & $\Rightarrow$ & $m$ & $\boldsymbol{v}$ & 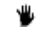 & $\|$ & $\Leftarrow$ & $\pi$ & $\pi$ & $\Rightarrow$ \\
\hline 0 & 523 & 782 & 814 & 815 & 836 & 839 & 840 & 840 & 849 & 855 \\
\hline$M$ & $\pi$ & $\Rightarrow$ & $v$ & $n$ & $m$ & $r$ & $\pi$ & $t$ & $\boldsymbol{*}$ & $\star$ \\
\hline 0 & 543 & 657 & 752 & 762 & 779 & 780 & 795 & 795 & 815 & 837 \\
\hline$n$ & $m$ & $h$ & $m$ & $n$ & $\approx$ & 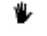 & 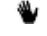 & 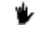 & 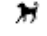 & $\star$ \\
\hline 0 & 596 & 616 & 650 & 717 & 763 & 766 & 769 & 784 & 785 & 785 \\
\hline & $n$ & $\pi$ & $n$ & $n$ & $\Delta$ & 구 & ה & $\star$ & 4 & $\star$ \\
\hline
\end{tabular}

$\begin{array}{lllllllllll}0 & 269 & 270 & 272 & 333 & 442 & 636 & 647 & 650 & 669 & 721\end{array}$

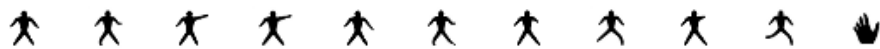
$\begin{array}{lllllllllll}0 & 259 & 260 & 333 & 515 & 578 & 644 & 708 & 720 & 727 & 750\end{array}$

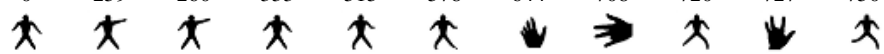

$\begin{array}{lllllllllll}0 & 94 & 260 & 270 & 399 & 499 & 672 & 695 & 698 & 702 & 704\end{array}$

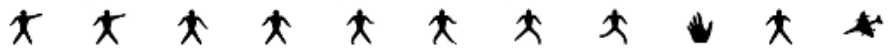
$\begin{array}{lllllllllll}0 & 94 & 259 & 272 & 384 & 488 & 664 & 691 & 699 & 701 & 709\end{array}$

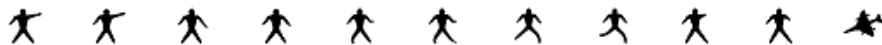

$\begin{array}{lllllllllll}0 & 230 & 269 & 384 & 399 & 487 & 498 & 510 & 515 & 523 & 713\end{array}$

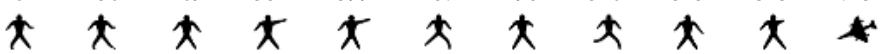
$\begin{array}{lllllllllll}0 & 230 & 346 & 383 & 442 & 459 & 481 & 488 & 499 & 578 & 700\end{array}$

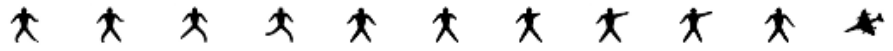
$\begin{array}{lllllllllll}0 & 95 & 383 & 449 & 476 & 510 & 669 & 691 & 695 & 734 & 750\end{array}$

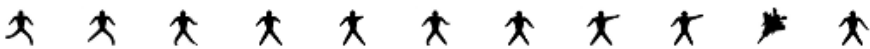
$\begin{array}{lllllllllll}0 & 95 & 346 & 452 & 481 & 487 & 647 & 664 & 672 & 720 & 721\end{array}$

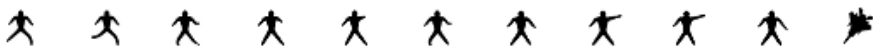

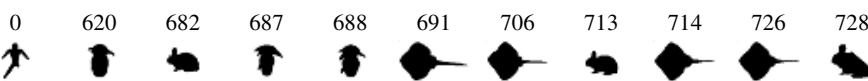

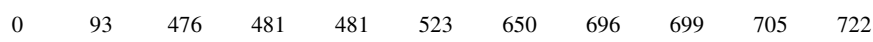

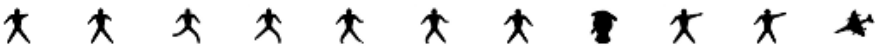

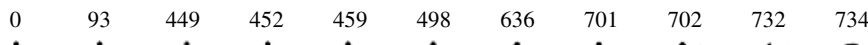

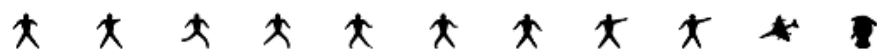

Fig. 5. Classes 2 and 3

visually be explained: A coarse plane "looks" more like a fish with two big fins than a very detailed plane.

Next, this approach is used on the data base used by Sebastian et al. 16]. This data base contains 9 classes with 11 shapes each. Some of the shapes are 
occluded or deformed versions of another shape in the class. Just as in [16], a score $D^{*}\left(S_{1}, S_{2}\right)$ is set to be a non-negative number, ranging towards 1000 . This is achieved by taking (recall Eq. 3)

$$
D^{*}\left(S_{1}, S_{2}\right)=1000\left(1-D\left(S_{1}, S_{2}\right)\right)
$$

Now 0 denotes a perfect match and values towards 1000 a random match. The results per class are shown in Figs. 4,8. We have chosen to show all results, as this better reveals the potential of matching methodology.

In each of the figures, the first column resembles the shape matched with itself, resulting in a score of 0 . The next 10 columns give the second to eleventh best match. Ideally, this would be shapes from the same class. The score of each shape is taken as an eleven dimensional vector with each value being zero or one. A one at position $i$ denotes a shape at the $i^{\text {th }}$ position that belongs to the same class, while a zero denotes a shape of a different class. The total class score is then given as the sum of the eleven vectors in the class, ideally being a vector containing 11 elevens. Table 1 gives these results.

\section{Discussion of Results}

Table 1 shows that some classes (6 and 9) yield a perfect score. Other classes contain matchings to objects of other classes. For some this occurs at higher positions, but in three cases already the second best match is wrong.

All these cases are caused by the choice of the matching algorithm, the removal of equidistant points. This is strongest visible in the third class, bottom of Fig. 5. The $9^{t} h$ row introduces a shape that has a large occlusion. This relates to removing a set of neighbouring points along the shape instead of the taken approach. An indication that "something is wrong" is given by the high cost for the second best match (620), compared to the other second best matches in this class $(\leq 269)$. Is introduces a complete row of wrong matches.

A similar effect, albeit in the opposite way, occurs in the fifth class, bottom of Fig. 6. The third row shows an occluded hand, which relates to a local addition of a set of neighbouring points along the shape. Again a high cost for the second best match is obtained. Assuming only equidistant removal of points, however, the second best match is visually correct. The fingers correspond to the four legs of the cow, while the blown-up thumb relates to the cow's head and body. The same thing can be said about the occluded rabbit in class 8, top of Fig. 8 .

Obviously, the human classification is not perfectly mimicked by the algorithm. The total amount of errors compared to the human observer classification is given by $(0,3,6,6,12,17,24,28,42,43,58)$. If the three most clear occlusioncaused outliers are left out, this is $(0,0,3,5,9,15,22,25,39,41,56)$.

As a way to avoid the removal of points in one of the sign of inner product diagram, one can obtain a parameterisation of exactly $n$ points. This results in more or less the same outcome, since it still does not take into account the effects of occlusion. Secondly, forcing a standard number of points along the 


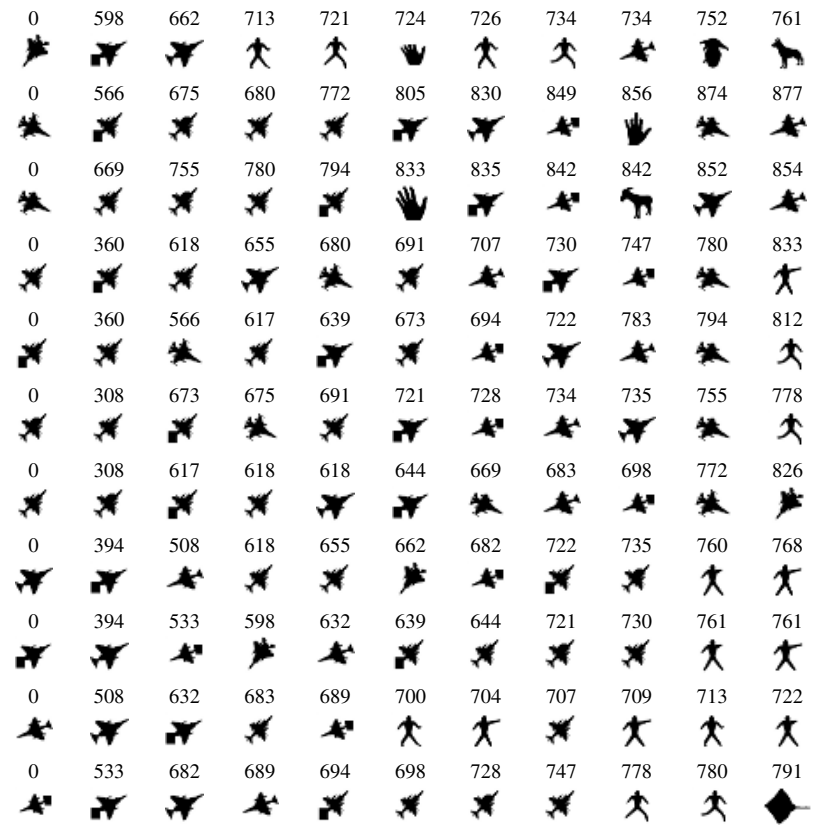

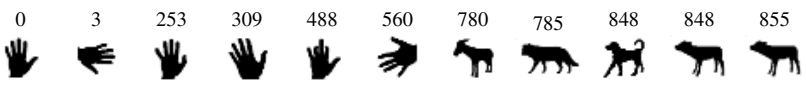
$\begin{array}{lllllllllll}0 & 304 & 309 & 358 & 443 & 646 & 763 & 795 & 798 & 818 & 825\end{array}$

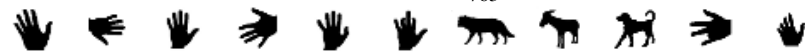
$\begin{array}{lllllllllll}0 & 842 & 900 & 902 & 916 & 917 & 918 & 954 & 965 & 969 & 969\end{array}$

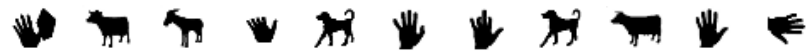
$\begin{array}{lllllllllll}0 & 630 & 715 & 724 & 756 & 758 & 761 & 772 & 780 & 787 & 805\end{array}$ "y $\begin{array}{lllllllllll}0 & 614 & 644 & 681 & 698 & 710 & 721 & 743 & 769 & 772 & 786\end{array}$

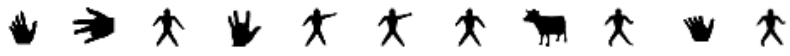
$\begin{array}{lllllllllll}0 & 358 & 560 & 562 & 591 & 694 & 737 & 781 & 782 & 840 & 849\end{array}$

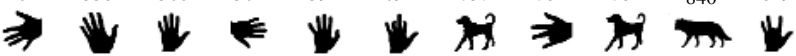
$\begin{array}{lllllllllll}0 & 3 & 254 & 304 & 488 & 562 & 779 & 786 & 847 & 848 & 855\end{array}$

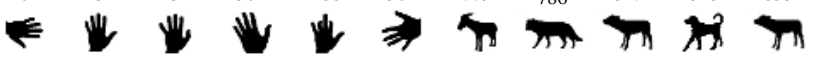
$\begin{array}{lllllllllll}0 & 253 & 254 & 443 & 496 & 591 & 809 & 842 & 862 & 883 & 897\end{array}$

U $\begin{array}{lllllllllll}0 & 488 & 488 & 496 & 646 & 694 & 795 & 807 & 827 & 839 & 848\end{array}$

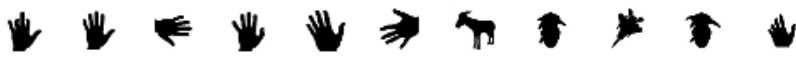
$\begin{array}{lllllllllll}0 & 334 & 630 & 681 & 727 & 783 & 789 & 789 & 802 & 803 & 809\end{array}$ $v \Rightarrow \omega * t \star t \star \omega t$

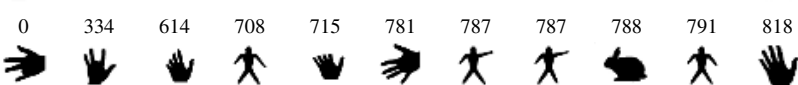

Fig. 6. Classes 4 and 5 


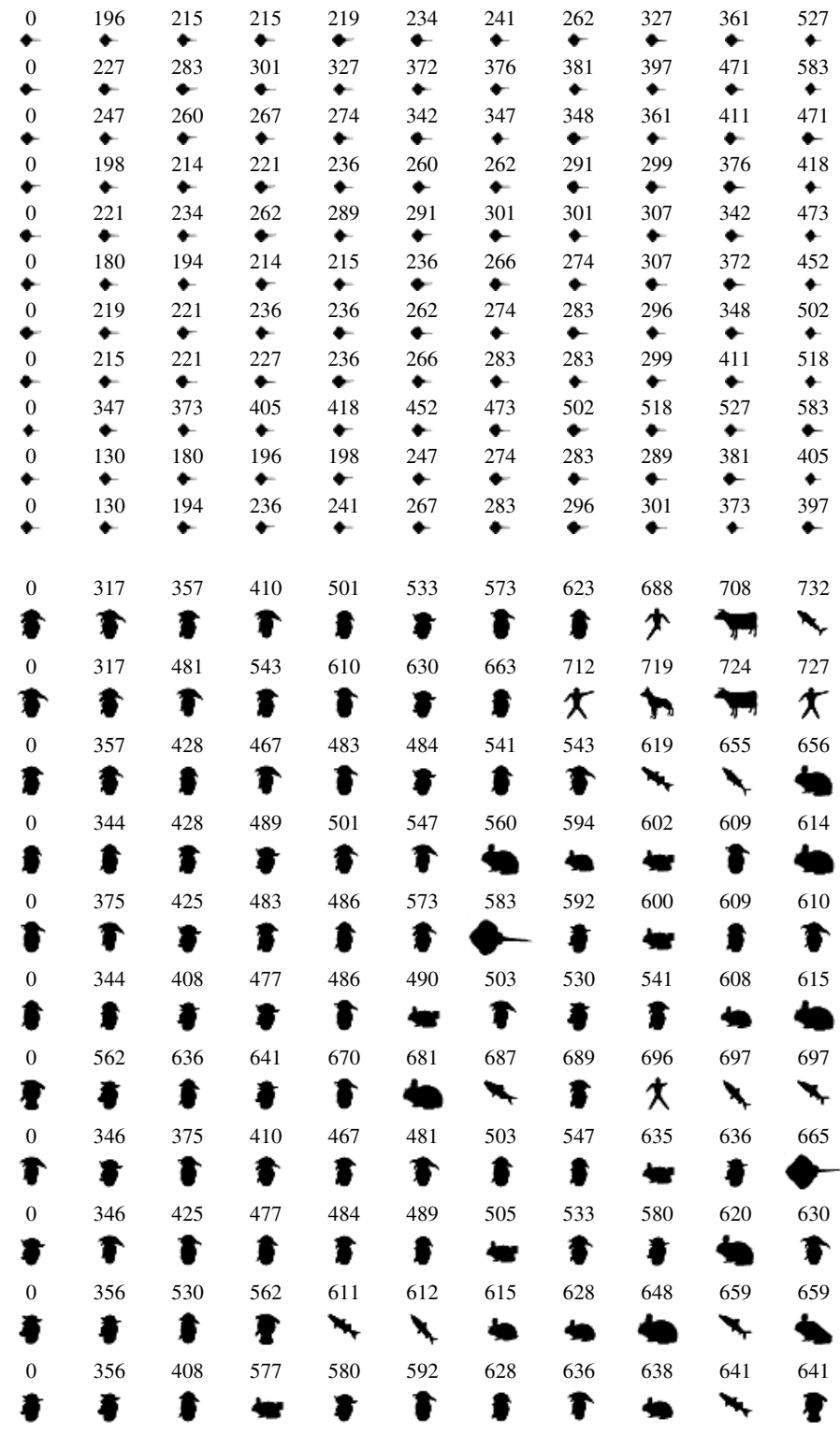

Fig. 7. Classes 6 and 7

shape wipes out the complexity of shape, so the matching actually yields worse results.

First attempts have been made in order to remove a set of $\frac{m}{n_{2}}$ locally neighbouring points. For the occluded human figure, this yielded a better matching to other human shapes. It is, however, computationally very expensive implemented. To compare two shapes takes approximately tens of minutes, compared 


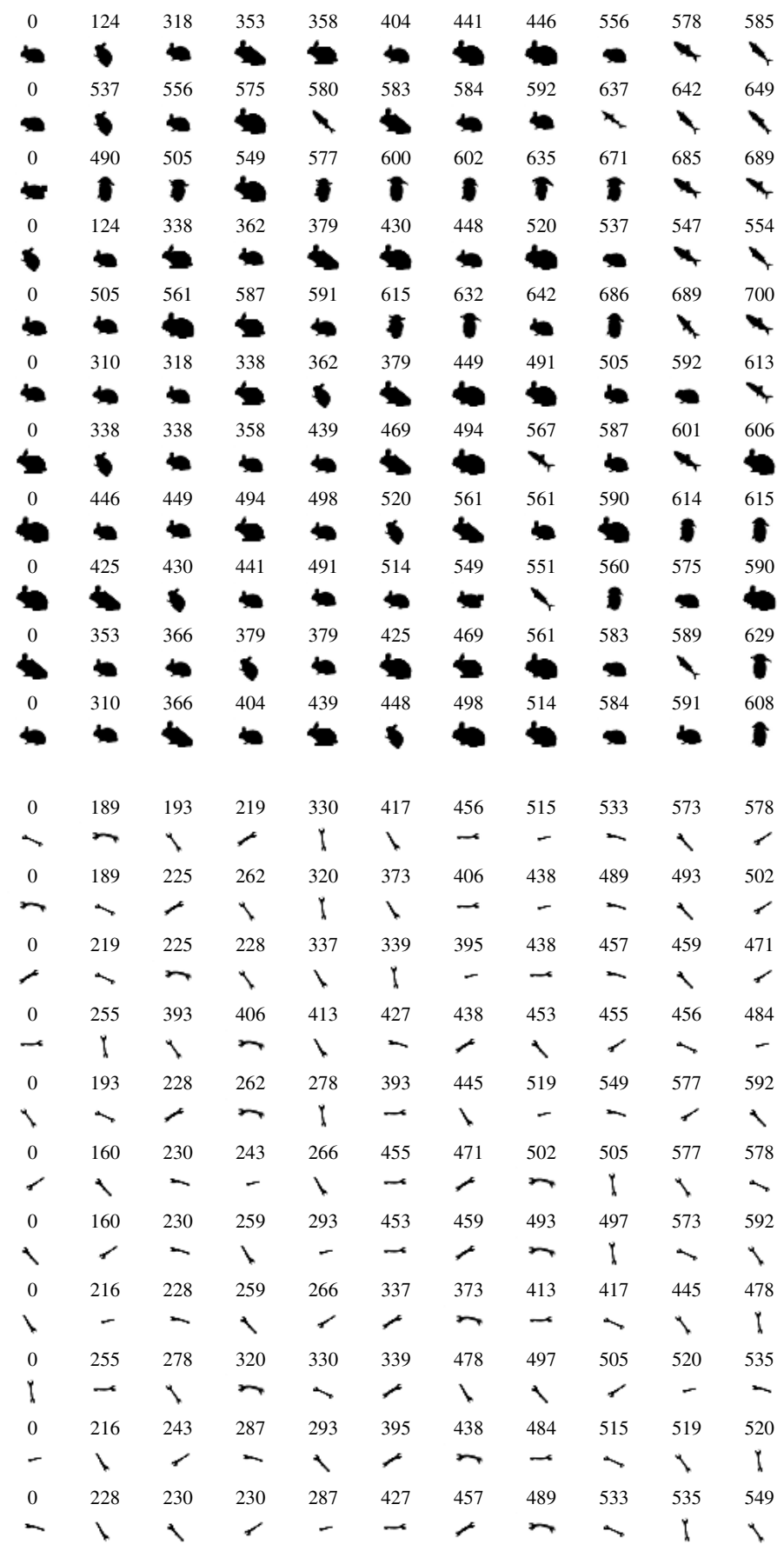

Fig. 8. Classes 8 and 9 
to several seconds in the equidistant case. However, as the optimal match is a summation of a set of multiplications, a fast dynamic program may be available. In this case the task would be to find a shortest manifold in $4 \mathrm{D}$.

\section{Summary and Conclusions}

A new shape descriptor is introduced. It is based on pairs of points on the shape that lie on a circle that is tangent to the shape at these points. It is therefore closely related to both Medial Axis and Symmetry Set methods. Each point on the shape is compared to all other points on the shape regarding a geometrical relation. Based on this, to each pair of points a value +1 or -1 is assigned. This yields an efficient data structure.

Secondly, shapes can be compared using this data structure. As test, a general data base 16 was used, containing shapes in different classes. Some of the shapes are severely occluded. To compare two data structures, the used approach removed a set of equidistant points along the shape, thus enforcing two shapes parameterised with the same number of points. This allows simple comparison of two data structures.

Although this matching assumption is very general and a priori not suited for occluded shapes, results were relatively good. The comparison of two shapes can be done in few seconds, using non-optimised Mathematica code. Some shape classes were completely correct classified, while other had a correct score for most of the shapes. The shapes that significantly scored bad were shapes with a large blocked occlusion, or with a locally removed part. These parts cannot be matched correctly by definition with the used method. We note that these deformed shapes give a relatively simple different Medial Axis. Secondly, we only matched one shape to another, allowing the changes to appear in only one shape. In general, the matching involves changes to both shapes, for example in matching the hands of class 5 (see Fig. 6, bottom) with different occluded fingers.

An obvious amendment of the matching algorithm is the possibility of removing a set of neighbouring points. This will solve the problem of occluded parts, both where a part of the shape is removed, and where a part (a block) is added. Second, the method is to be designed to find the optimal solution allowing both data structures to be changed. As the optimal match is a summation of a series of multiplications, a fast shortest-path based dynamic program may be available to incorporate these two amendments simultaneously.

\section{References}

1. Blum, H.: Biological shape and visual science (part i). Journal of Theoretical Biology 38 (1973) 205-287

2. Kimia, B.: On the role of medial geometry in human vision. Journal of Physiology - Paris 97 (2003) 155-190

3. Ogniewicz, R.L., Kübler, O.: Hierarchic voronoi skeletons. Pattern Recognition 28 (1995) 343-359 
4. Sebastian, T., Kimia, B.B.: Curves vs. skeletons in object recognition. Signal Processing 85 (2005) 247-263

5. Siddiqi, K., Kimia, B.: A shock grammar for recognition. Proceedings CVPR '96 (1996) 507-513

6. Sebastian, T., Klein, P., Kimia, B.B.: Recognition of shapes by editing shock graphs. IEEE Transactions on Pattern Analysis and Machine Intelligence 26 (2004) 550-571

7. Pelillo, M., Siddiqi, K., Zucker, S.: Matching hierarchical structures using association graphs. IEEE Transactions on Pattern Analysis and Machine Intelligence 21 (1999) 1105-1120

8. Bruce, J.W., Giblin, P.J., Gibson, C.: Symmetry sets. Proceedings of the Royal Society of Edinburgh 101 (1985) 163-186

9. Giblin, P.J., Kimia, B.B.: On the local form and transitions of symmetry sets, medial axes, and shocks. International Journal of Computer Vision 54 (2003) $143-156$

10. Blake, A., Taylor, M., Cox, A.: Grasping visual symmetry. Proceedings Fourth International Conference on Computer Vision (1993) 724-733

11. Blake, A., Taylor, M.: Planning planar grasps of smooth contours. Proceedings IEEE International Conference on Robotics and Automation (1993) 834-839 vol.2

12. Kuijper, A., Olsen, O.: On extending symmetry sets for $2 \mathrm{D}$ shapes. In: Proceedings of S+SSPR. (2004) 512-520 LNCS 3138.

13. Kuijper, A., Olsen, O., Giblin, P., Bille, P., Nielsen, M.: From a 2D shape to a string structure using the symmetry set. In: Proceedings of the 8th European Conference on Computer Vision. Volume II. (2004) 313-326 LNCS 3022.

14. Kuijper, A., Olsen, O.: Transitions of the pre-symmetry set. In: Proceedings of the 17th International Conference on on Pattern Recognition. Volume III. (2004) 190-193

15. Sharvit, D., Chan, J., Tek, H., Kimia, B.: Symmetry-based indexing of image databases. Journal of Visual Communication and Image Representation 9 (1998) $366-380$

16. Sebastian, T., Klein, P., Kimia, B.B.: Recognition of shapes by editing shock graphs. In: Proceedings of the 8th ICCV. (2001) 755-762 